\title{
Have SPECT artifacts breathed their last?
}

\author{
Christopher L. Hansen, MD \\ a Jefferson Heart Institute, Thomas Jefferson University, Philadelphia, PA \\ Received Jan 27, 2016; accepted Jan 27, 2016; Published online February 18, 2016 \\ doi: $10.1007 / \mathrm{s} 12350-016-0428-0$
}

\section{See related article, pp. 698-707}

Everything should be made as simple as possible, but not simpler-Albert Einstein

The ability to reconstruct a 3-dimensional distribution from its projections ranks among the greatest breakthroughs in medical imaging. The consequent revolution has transformed x-ray, nuclear imaging and is even making inroads in echocardiography. At the same time, this revolution has introduced a host of new artifacts that have befuddled, confused and all to often, misled physicians interpreting these scans. Although there are multiple potential sources of artifact, soft tissue attenuation and patient motion are the most important.

Soft tissue attenuation is more problematic in nuclear imaging. Whereas $\mathrm{x}$-ray imaging is based on the differential attenuation of various tissues and organs, nuclear imaging is based on the differential uptake of an isotope between normal and diseased tissues. Since soft tissue attenuates photons from $\mathrm{x}$-rays or radioisotopes similarly the property being measured and reconstructed in $\mathrm{X}$-ray computed tomography becomes a source of artifact for SPECT. The 1990s saw the introduction of simultaneous transmission scanning and iterative reconstruction techniques that can incorporate and correct for attenuation which have shown great promise in correcting for this artifact and improving accuracy. ${ }^{1}$

Motion can be broken into two broad categories. The first we will call translational, this is where the patient physically moves. This artifact is usually minimal in a cooperative patient and there are useful software corrections that can reduce or eliminate the

Reprint requests: Christopher L. Hansen, MD, Jefferson Heart Institute, Thomas Jefferson University, 925 Chestnut St, Mezzanine,

Philadelphia, PA, 19107; christopher.hansen@jefferson.edu J Nucl Cardiol 2017;24:708-710.

1071-3581/\$34.00

Copyright (c) 2016 American Society of Nuclear Cardiology. artifact in the patient who cannot remain still during imaging. ${ }^{2}$ Up and down motion is most easily corrected, side to side is more difficult but achievable and, unfortunately, rotational motion has not yet been successfully addressed.

The second category of motion, which we will call periodic, includes both the beating of the heart and motion due to respiration. Electrocardiographic gating is now routinely used. Although this is not primarily employed for reducing artifact, it has contributed greatly to the utility of myocardial perfusion imaging by allowing the evaluation of wall motion and ejection fraction. Respiratory gating has been proposed and explored many times but has not yet achieved widespread clinical application. ${ }^{3,4}$

CT, echocardiography, and MRI have high enough temporal resolution that imaging can frequently be done in a single breath hold. This is not an option in nuclear due to longer imaging times, necessitated by lower doses of radiation. This requires list mode acquisition and/or respiratory gating but also an accurate understanding and modeling of the pattern of respiration.

Chrysanthou-Baustert et al report the results of an ambitious undertaking in this current Journal. They study periodic motion by designing a complex phantom that models both cardiac contraction and respiratory motion. ${ }^{5}$ This model incorporates translation of the heart along a caudal-cephalad axis, tidal expansion of lungs made of a less attenuating material to mimic expanding lungs and a cardiac chamber that expands and contracts. The heart was made of concentric silicone membranes that can stretch and shrink to simulate systole and diastole, a refinement of a rubber membrane-based model they reported previously. ${ }^{6}$ The phantom was imaged modeling shallow and deep breathing and again after inserting small and large perfusion defects. As expected, attenuation was most pronounced in the basal segments representing areas deepest inside the thorax and attenuation correction was helpful in reducing this artifact. They report that respiratory motion tended to produce artifacts centered on the anterior and inferior walls. The clinical impact was minor for severe defects 
but more significant for small ones. So, does this model now cover all possible artifacts? Could respiratory gating finally allow for clinicians to eliminate this source of artifact?

There are many patterns of breathing, the most common in healthy adults are costal (breathing primarily by expansion of the chest) and diaphragmatic (breathing primarily by lowering the diaphragm). Diaphragmatic breathing is associated with a more "relaxed" physiologic state-in fact most systems of meditation teach it. In most patients breathing is a mixture of both but tends more toward costal when the patient is anxious or in discomfort. It is not uncommon for patients to have predominantly costal breathing at the outset then transition to diaphragmatic breathing as they habituate and begin to relax. Patient positioning (supine versus prone versus upright) can also influence the type of breathing as can the use of accessories (e.g., chest or abdominal straps). Some investigators have noted that raising the arms above the head (which is typical in the majority of SPECT imaging) tends to reduce diaphragmatic excursion with respiration. ${ }^{7}$ Respiratory patterns can also vary in different disease states. For example, patients with COPD and hyper-expanded lungs will have much lower diaphragmatic excursion during respiration than normals. Imaging patients shortly after vigorous exercise (which is essential when using isotopes that redistribute quickly, such as 201-Thallium) can produce a gradual upward motion of the heart (termed "upward creep") that occurs with decreasing tidal volumes as the patient catches his breath. ${ }^{8,9}$

Further complicating the issue is the fact that the heart not only translates during respiration it rotates as well. ${ }^{7}$ This is most likely due to the fact that the apex is free, whereas the base of the heart is fixed. This pattern can vary from patient to patient as well as from breath to breath. Other investigators have reported what they term hysteresis: for the same diaphragmatic position, the heart is in a different location depending on whether the patient is in inspiration versus expiration. ${ }^{10}$ Therefore, the pattern of respiration may vary from patient to patient, patient positioning but also may change in a given patient during image acquisition. Accurately correcting for respiratory motion will be critically dependent on using the correct model. When a patient's breathing pattern is different from the model or changes during image acquisition, the risk for introducing new artifacts is significant.

Therefore, it seems that completely accurate modeling of the heart with respiration is a herculean task and may be impossible to achieve reliably. But then it is not to say that it is completely futile. Even partial correction for respiratory artifact might improve the accuracy of MPI and make it worth pursuing as long as the clinician remains alert to the problem of introducing artifacts if the model's underlying assumptions about the pattern of respiratory motion are incorrect.

One insight more likely to result in clinical benefits is the appreciation that problems with misregistration between transmission and emission scans when employing attenuation correction can be influenced by the temporal resolution of the transmission scan. Fast CT-based transmission scans will freeze the heart and lungs and one particular position, whereas slower CT or scanning line source-based systems blur the relation since they are acquired over several breaths. These latter systems appear to work better. ${ }^{11}$ The authors used a single slice Hawkeye CT. Though they did not report acquisition time, it is more in the 20-30 s range for this system implying that there would be enough blurring to minimize misregistration artifact.

So what are we to make of these results? The results reported by Chrysanthou-Baustert et al are somewhat reassuring in that the severe perfusion defects were routinely detected, despite the presence of rather complicated artifacts. That is, the highest risk patients should still be correctly identified and the prognostic power of MPI (which is its most clinically useful feature) remains intact. $^{12}$ This is not to neglect the importance of diagnostic accuracy; we should continue to pursue all avenues that might improve this. It is helpful that the artifacts encountered are usually mild and appear to be atypical for coronary distributions.

So does this paper mean that we will soon have a solution to all SPECT artifacts? These data are helpful, but let's not hold our breath.

\section{References}

1. Corbett JR, Ficaro EP. Clinical review of attenuation-corrected cardiac SPECT. J Nucl Cardiol 1999;6:54-68.

2. Matsumoto N, Berman DS, Kavanagh PB, Gerlach J, Hayes SW, Lewin HC, et al. Quantitative assessment of motion artifacts and validation of a new motion-correction program for myocardial perfusion SPECT. J Nucl Med 2001;42:687-94.

3. Cho K, Kumiata S, Okada S, Kumazaki T. Development of respiratory gated myocardial SPECT system. J Nucl Cardiol 1999; 6:20-8.

4. Zubal IG, Bizais Y, Bennett GW, Brill AB. Dual gated nuclear cardiac images. IEEE Trans Nuclear Sci 1984;31:566-9.

5. Chrysanthou-Baustert I. et al. Characterization of attenuation and respiratory motion artifacts and their influence on SPECT MP Image evaluation using a dynamic phantom assembly with variable cardiac defects. J Nucl Cardiol 2016.

6. Chrysanthou-Baustert I, et al. Diagnostic sensitivity of SPECT myocardial perfusion imaging using a pumping cardiac phantom with inserted variable defects. J Nucl Cardiol 2013;20:609-15.

7. Bogren HG, Lantz BM, Miller RR, Mason DT. Effect of respiration on cardiac motion determined by cineangiography. Implications concerning three-dimensional heart reconstruction using computer tomography. Acta Radiol Diagn (Stockh) 1977;18:609-20. 
8. Friedman J, Van Train K, Maddahi J, Rozanski A, Prigent F, Bietendorf J, et al. "Upward creep" of the heart: A frequent source of false-positive reversible defects during thallium-201 stress-redistribution SPECT. J Nucl Med 1989;30:1718-22.

9. Mester J, Weller R, Clausen M, Bitter F, Henze E, Lietzenmayer $\mathrm{R}$, et al. Upward creep of the heart in exercise thallium 201 single photon emission tomography: Clinical relevance and a simple correction method. Eur J Nucl Med 1991;18:184-90.
10. Nehrke K, Börnert P, Manke D, Böck JC. Free-breathing cardiac MR imaging: Study of implications of respiratory motion-initial results. Radiology 2001;220:810-5.

11. Konik A, Kikut J, Lew R, Johnson K, King MA, et al. Comparison of methods of acquiring attenuation maps for cardiac SPECT in the presence of respiratory motion. J Nucl Cardiol 2013;20:1093-107.

12. Hansen CL. The prognosis for prognosis remains excellent. J Nucl Cardiol 2013;20:501-3 